\title{
INKJET PRINTING OF 3D NANO-COMPOSITES FORMED BY PHOTOPOLYMERIZATION OF AN ACRYLATE MONOMER
}

\author{
Casey Check, Richard Chartoff *, Sean Chang \\ University of Oregon, Materials Science Institute, \\ Department of Chemistry and Biochemistry, Eugene, Oregon 97405, USA \\ * Contact: Chartoff@uoregon.edu
}

\begin{abstract}
This paper considers inkjet printing of optical quality 3D nano-composites from diethylene-glycol diacrylate monomer (DEGDA) containing $\mathrm{ZrO}_{2}$ nanoparticles at varying concentrations. One application for the composites is for gradient refractive index (GRIN) lenses. The process involves printing of a nanoparticle loaded monomer "ink" onto a substrate and then photopolymerizing the monomer layer by layer using UV light. The results of the study confirm that the presence of nanoparticles favorably affects the reaction kinetics. The reaction rate and chemical conversion are enhanced considerably by the nanoparticles. A seamless interface between the layers, which are $20 \mu \mathrm{m}$ thick, may be achieved if the conversion level of the layer onto which ink is deposited is limited at 60 to $80 \%$. Dynamic mechanical analysis (DMA) data indicate that both the glass transition and sub $\mathrm{Tg}$ viscoelastic properties are influenced by the nanoparticles. When nanoparticles are introduced the Tg relaxation shifts to lower temperatures and a sub $\mathrm{Tg}$ relaxation appears whose intensity increases with particle concentration. These results are consistent with a molecular confinement model involving a lower crosslink density rubbery layer at the polymer-particle interfaces.
\end{abstract}

Key words: 3D printing of nano-composites, particle filled nano-composites, nanoparticle confinement effects, polymeric GRIN lenses, $\mathrm{ZrO}_{2}$ nano-composites

\section{INTRODUCTION}

Inkjet printing is a convenient method for fabricating gradient polymer matrix nanocomposites, and has been intensively studied by our research group at the University of Oregon. Such a route towards manufacture of these composites involves printing of a nanoparticle loaded monomer "ink" onto a substrate and then photopolymerizing the monomer layer by layer using UV light. The process is a variation of 3-D printing. However, over-curing each of the layers leads to a defect structure with a laminated discrete layer morphology in the material. The interfaces between the layers are defects which compromise the desirable mechanical, physical, and optical properties of the composite. Precisely controlling the extent of cure of each of the layers can ensure a seamless bulk material free from such defects.

We have been considering formation of optical quality nano-composites from diethyleneglycol diacrylate monomer (DEGDA) containing $\mathrm{ZrO}_{2}$ nanoparticles at varying concentrations. One application for the composites is for gradient refractive index lenses. DEGDA polymers are optically transparent to visible light. If the nanoparticles are well dispersed in the monomer they effectively modify the refractive index in proportion to their volume concentration. They will not interfere with transmission of light, since they are much smaller than the wavelength of light. In our process photocuring is accomplished in an inert atmosphere by exposing the monomer/nanoparticle mixture to UV radiation delivered by a focused LED emitting at $365 \mathrm{~nm}$. Both the time duration of exposure and the UV light intensity determine the degree of conversion. In general, the exposure time duration is fixed and the light intensity may be varied. In our inkjet printer each element of monomer is exposed to UV radiation for $\sim 1$ second. This study explores the effects of varying the LED intensity, as well as $\mathrm{ZrO}_{2}$ nanoparticle loading on the extent of cure reaction within specific DEGDA / $\mathrm{ZrO}_{2}$ systems. We use these data to determine the appropriate light exposure to ensure that seamless composites are formed, since 
irregularities at the layer interfaces will interfere with light transmission through the composites. Additional reference to kinetics of photopolymerization of nanofilled DEGDA can be found in a recent publication [ 1 ]. In addition we have applied for a US patent on various aspects of this work [ 2 ]. We are not aware of other publications of research on nanofilled DEGDA.

Drop on demand (DOD) printing allows for precise and controlled delivery of material onto a substrate. Our printer operates via piezoelectric deposition. In the inkjet nozzle, the fluid is held in place via surface tension, and drop formation is induced via a pressure wave generated as a result of constriction of a piezoelectric element within the nozzle. The drop volume is 10 picoliters, which makes possible composition resolution at the micron level.

\section{Forming polymer networks by photoreaction:}

Network polymers formed by UV photocuring are a class of thermosets whose reaction and thermal characteristics can be characterized by thermal analysis methods, particularly differential scanning calorimetry (DSC), dynamic mechanical analysis (DMA) and Thermalmechanical analysis (TMA). Commonly photopolymers are applied as films or surface coatings. They are most often formed from the reaction of multifunctional, unsaturated acrylate or methacrylate monomers by a free radical mechanism. The reactions are facilitated by soluble photoinitiators added in small amounts to the monomers. The photoinitiators are light sensitive compounds that decompose on irradiation to generate the free radicals that then initiate the polymerization. The initiating radicals combine with available monomer forming primary radicals that subsequently propagate through additional monomer units to create high molecular weight polymers.

\section{Photopolymers may be heterogeneous with more than one phase present even when only one monomer is involved. Because of this heterogeneity they have unusually broad glass transitions:}

In a single component system which crosslinks such as DEGDA, phase heterogeneity results from the statistical nature of the chain polymerization process and the random coil conformation of the growing chains, as well as the increased reactivity of dangling acrylate groups arising from 1,2 reactions of monomer units already attached to the network. This is consistent with the percolation model forwarded by Kloosterboer et al. [ 3-6]. Simulation data [3] indicate that for a single monomer both low and high cross-link density regions develop even at very low conversions. The high-density phase consists largely of fairly rigid cross-linked gel material and the low-density phase is monomer swollen lesser crosslinked gel.

Experimental time lapse dynamic mechanical data are consistent with this model. They indicate that the amount of the high-density phase increases as the polymerization proceeds [7]. This phase has a high $\mathrm{Tg}$, while the low density phase has a low $\mathrm{Tg}$. These data also show that the two distinct loss dispersions shift as the degree of conversion advances. The low temperature loss dispersion advances in temperature, is progressively reduced in intensity, and is absorbed into the high temperature loss dispersion. Also the high temperature (major) dispersion shifts slightly to the right and increases in breadth. The major loss dispersion covers an unusually wide temperature range, indicating that the cross-link network is quite microheterogeneous. Additional data on phase heterogeneity during cure in such systems have been cited in other publications [7-10]. A review of the general characteristics of the photopolymerization reactions of diacrylates is provided in $[7,10]$.

\section{EXPERIMENTAL}

\section{Materials:}


Diethylene glycol diacrylate DEGDA was obtained from Sartomer and used as received without further purification. The photoinitiator employed was Irgacure 184 from BASF Chemicals. The photoinitiator concentration was $1.5 \mathrm{wt} \%$.<smiles>C=CC(=O)OCCOCCOC(=O)C=C</smiles>

Diethylene glycol diacrylate Nanoparticle characterization:<smiles>O=C(c1ccccc1)C1(O)CCCCC1</smiles>

Irgacure 184

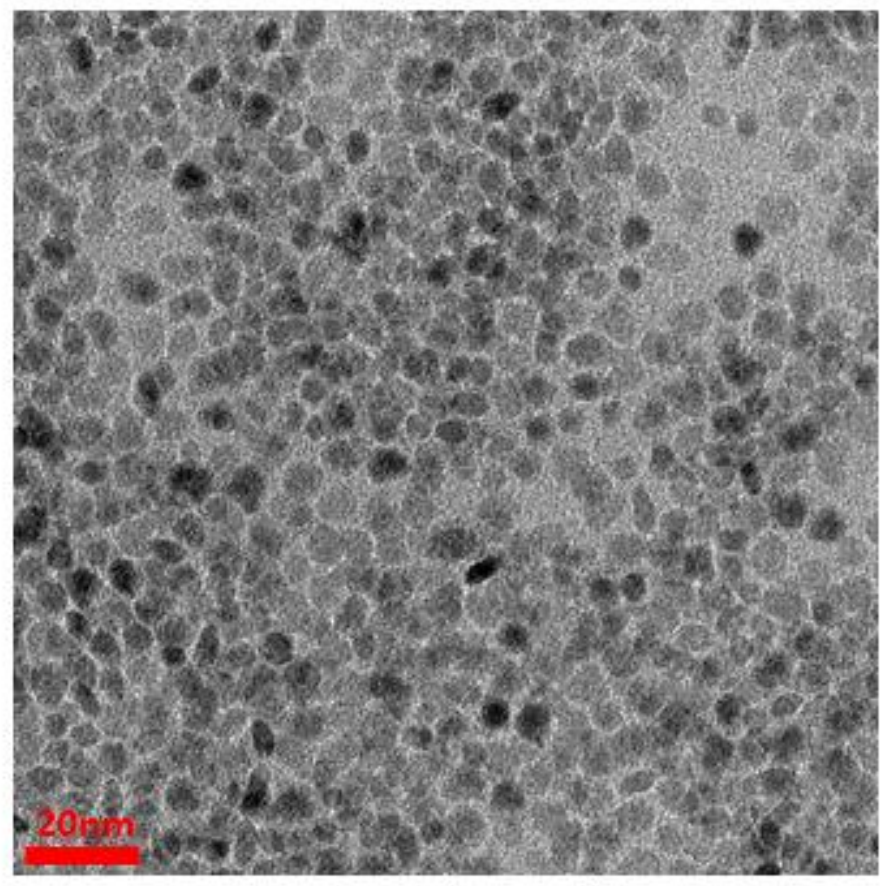

Figure 1 HRTEM image of $\mathrm{ZrO}_{2}$ nanoparticles used in this study; scale bar is $20 \mathrm{~nm}$

\section{Nanoparticles:}

Fig. 1 is a high resolution transmission electron microscopy (HRTEM) image of $\mathrm{ZrO}_{2}$ nanoparticles of this study. The average particle size is $7 \mathrm{~nm}$ and the particle sizes are quite uniform. The nanoparticles were obtained from Pixelligent-the ligand capping the particles consists of a silane as discussed in [11]. NMR analysis of the particles indicates that the ligand contains unreacted methacrylate groups. The amount of capping agent is $12.5 \%$ by weight as measured by thermogravimetric analysis (TGA). The scale bar at bottom left of the HRTEM photomicrograph is $20 \mathrm{~nm}$.

\section{Nanoparticle Inks:}

Inks containing colloidally suspended $\mathrm{ZrO}_{2}$ nanoparticles were prepared by simple mixing of the silane treated particles suspended in methylene chloride into DEGDA monomer and evaporating the methylene chloride as described in the following paragraph. The suspension viscosities were quite low and independent of particle concentration so that inkjet printing was not difficult. There is considerable precedent for inkjet printing of nanoparticle suspensions 
indicating that fluid viscosities are relatively unaffected up to rather high particle concentrations as long as the particles are well dispersed [ 12-15]

\section{Diethylene glycol diacrylate (DEGDA) photopolymerization study experimental procedure:}

Samples containing 10, 20, 25, and $30 \%$ wt $\mathrm{ZrO}_{2}$ nanoparticles were prepared using a stock solution of Irgacure 184 in DEGDA. The nanoparticles were initially received in propylene glycol monomethyl ether acetate (PGMEA) which was removed by precipitation of the particles into hexane followed by collection by centrifugation. The white solid obtained was dispersed in a small volume of methylene chloride, re-precipitated into hexane, and collected by centrifugation. This procedure was repeated 3 times. The solid was then dispersed in a small amount of methylene chloride and added to the DEGDA / Irgacure 184 solution and the solvent was then removed by rotary evaporation. The resulting DEGDA / $\mathrm{ZrO}_{2}$ suspensions were dispensed into weighed hermetic DSC pans by a micropipette $(\sim 3 \mathrm{mg})$ hermetic DSC pans (uncovered) and purged in the dark under nitrogen for 30 minutes before polymerization. The samples then were cured by exposure to $1 \mathrm{sec}$ of UV light from a small spot LED (described below) focused at a height of 1 inch above the sample. A series of samples at each concentration was exposed, to a different UV intensity. The UV intensity was varied from $0 \%$ to $100 \%$ of the total LED power in $10 \%$ increments and the samples were immediately sealed in the DSC pan for thermal scan DSC residual cure analysis.

To determine kinetic effects of the nanoparticles on the photoreaction of DEGDA to form polymer, a thermal DSC scan was done on the photocured samples to determine the degree of conversion. All samples were scanned at $10^{\circ} \mathrm{C} / \mathrm{min}$. from $40^{\circ} \mathrm{C}$ to $330^{\circ} \mathrm{C}$ to determine the residual reaction exotherm and percentage of reaction. The reaction enthalpies are the corrected values determined by first subtracting the mass of the nanoparticles. So the values refer to heats of reaction of the monomer portion of the total sample mass. The "residual" $\Delta \mathrm{H}_{\mathbf{s}}$ value from this was compared with a second thermal DSC scan conducted for neat monomer in an inert atmosphere. The $\Delta \mathrm{H}$ for the residual exotherm of the photocured sample was compared to that of the unreacted pure monomer $(629.4 \mathrm{~J} / \mathrm{g})$ using the following equation.

$$
\left[1-\frac{\Delta H_{s}(J / g)}{629.4(J / g)}\right] x 100=\% \text { Conversion }
$$

( Equation 1)

Where $\Delta \mathrm{H}_{\mathrm{S}}$ is the residual reaction exotherm of a photopolymerized sample.

\section{UV curing of DSC samples using an advanced compact LED:}

Photocuring in DSC pans was accomplished with a custom designed compact smallspot size LED device from Clearstone Technologies, Minneapolis, MN as shown in Fig. 2. The LED has a square shaped light-spot footprint as shown of $\sim 1.5 \mathrm{~mm}$ at a primary wavelength of $365 \mathrm{~nm}$. The total LED power at peak is $78 \mathrm{~mW}$, yielding a power/area of $3466 \mathrm{~mW} / \mathrm{cm}^{2}$. The dispersed halo power surrounding the spot is $\sim 1 / 100$ of the spot power, a level that is not sufficient to effect cure. The small spot LED gives precise control over both wavelength and radiation intensity delivered to the sample. It delivers light exposures of a single wavelength, with uniform intensity for a controlled time interval, so as to give each element exactly the same cure profile. The LED allows for small volume incremental light exposures, which aids stress relaxation and thus helps mitigate the effects of residual stresses in the films. The radiation dose imparted for curing can be adjusted by varying the power supplied to the LED, the height of the LED above the curing monomer and the time of exposure. 

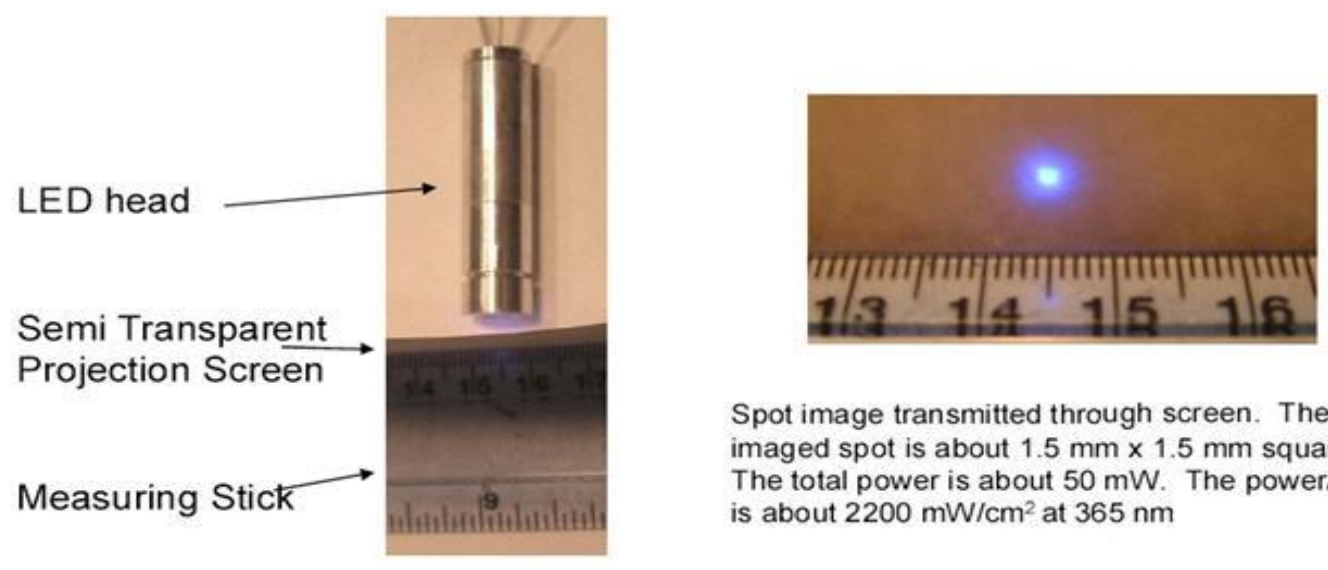

Spot image transmitted through screen. The imaged spot is about $1.5 \mathrm{~mm} \times 1.5 \mathrm{~mm}$ square.

The total power is about $50 \mathrm{~mW}$. The power/area is about $2200 \mathrm{~mW} / \mathrm{cm}^{2}$ at $365 \mathrm{~nm}$

Figure 2: Description of the small spot size UV LED used for photopolymerization

\section{DMA and TMA sample preparation and measurement procedure:}

Dynamic mechanical analysis (DMA) samples were prepared using open sided silicone rubber molds with both sides covered by glass slides; so as to minimize formation of bubbles in the part. Bubbles that formed were relocated towards the edge of the sample by tilting the cover slide slightly. Samples were polymerized with an LED array for 3 minutes on each side at $100 \%$ LED power. Sample size was $59 \times 13 \times 3 \mathrm{~mm}(\mathrm{~L} \times \mathrm{W} \times \mathrm{T})$. Samples were tested in single cantilever geometry in a TA Instruments Q800 DMA at a frequency of $1 \mathrm{~Hz}$ and a ramp heating rate of $4^{\circ} \mathrm{C} /$ minute. A small portion of each DMA bar was used for the TMA experiments. The LED array used for curing these specimens was a LH365-B device from Clearstone Technologies, Minneapolis, MN, which delivers a nominal intensity of $210 \mathrm{~mW} / \mathrm{cm}^{2}$ at $365 \mathrm{~nm}$. The device consists of an array of 18 LEDs which cover an area of $20 \mathrm{~cm}^{2}$. The degree of conversion for each sample was at least $90 \%$.

\section{Inkjet printing of composites:}

Gradient composite films were formed by inkjet printing in an $\mathrm{N}_{2}$ atmosphere. The printer used was a Dimatix Materials Printer, DMP-2800, manufactured by Fujifilm-Dimatix. The DMP is a laboratory research printer that enables the evaluation of ink jetting technology for new materials manufacturing and analytical processes. It is designed for carrying out "proof of concept" and development work with sophisticated capabilities for optimizing process parameters for a given application. The DMP is PC-controlled and has a substrate scanning "ink jet" deposition system with a visual drop observation camera, and spot location capability. It prints with user-fillable piezo-based jetting cartridges, each with 16 square nozzles spaced at $254 \mu \mathrm{m}$ such that ink drop volumes are 10 picoliters. It is designed specifically for working with organic fluid based "inks" and is equipped with a capability for nozzle and substrate heating up to $70^{\circ} \mathrm{C}$.

In our process monomer was printed onto a glass substrate to form layers $20 \mu \mathrm{m}$ in thickness and then reacted to form composites. Composites were multilayered -- 15 layers. Each layer was partially polymerized so that the free surface under the current layer was about $70 \%$ reacted. The layer immediately under the top layer further reacted as the top layer was polymerized. 


\section{EXPERIMENTAL RESULTS}

\section{DSC photopolymerization experiments:}

The results for the DSC photopolymerization experiments are shown in Fig. 3. Each data point represents an average value from a minimum of 3 separate dynamic DSC thermal scans with a variation of $+/-3 \%$. Clearly the cure advancement for a given light dose is greater for samples containing nanoparticles. There may be two possible explanations for this. First of all $\mathrm{ZrO}_{2}$ may act as a catalyst. However, the surface of our particles has been passivated by a silane ligand. A more likely explanation then relates directly to the silane coatings on the particles. Silanes are known to react with vinyl monomers and may influence the yields and alter the molecular weights achieved in polymerization [16, 17]. NMR analysis of the nanoparticles indicated that a vinyl silane is present which furthers this explanation. Keeping in mind that in these nanoparticle systems there is a very large dispersed particle surface area in contact with the monomer, the effect of the surface ligand should be quite significant. The limiting $90 \%$ conversion level achieved in these experiments is governed by vitrification. We determined that when the samples are exposed to $100 \%$ radiation intensity for longer time periods, such as 5 to 20 minutes, the conversion will be advanced closer to $100 \%$.

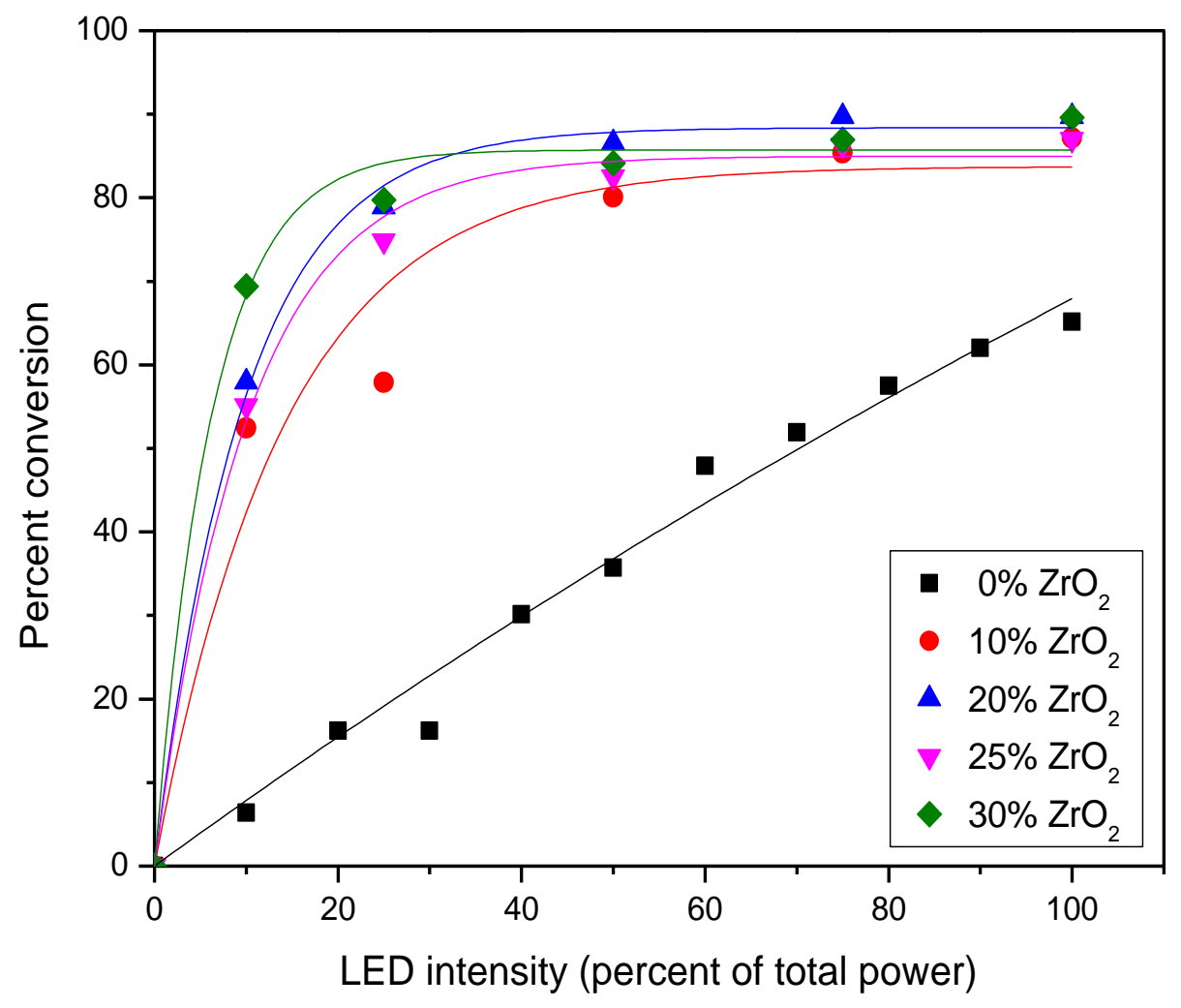

Figure 3: Reaction conversion percent as a function of LED intensity for various $\mathrm{ZrO}_{2}$ levels 


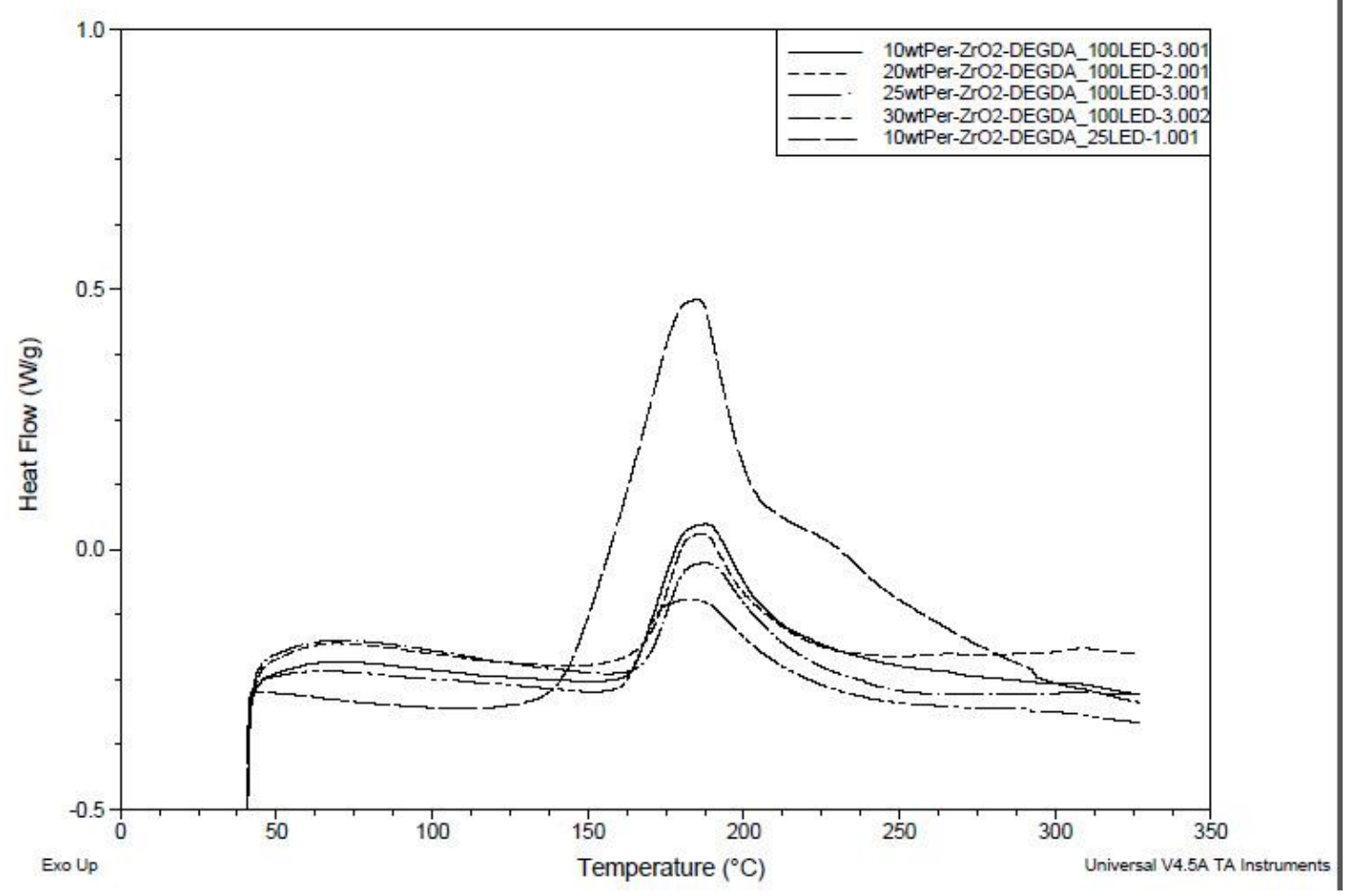

Figure 4: Composite plot of DSC curves for several compositions of monomer with nanoparticles exposed to $100 \%$ UV compared to one sample exposed to $25 \%$ UV

Fig. 4 is an overlay composite plot of DSC thermograms for several samples of DEGDA containing various concentrations of nanoparticles, each exposed to high intensity UV at $100 \%$ of source output. Also shown is the curve for a single sample exposed to much lower (25\%) UV intensity. The residual exotherm areas are indicative of the inverse of the amount of cure conversion for each sample, noting that some of the diminished exotherm results from an increase in nanoparticle content. The peak exotherms for these samples center around $190^{\circ} \mathrm{C}$. The sample exposed to $25 \%$ UV intensity shows a distinct shoulder at $\sim 220^{\circ} \mathrm{C}$, indicating a separate reaction mechanism. $220^{\circ} \mathrm{C}$ is where the thermal cure occurs for neat DEGDA monomer samples that do not contain nanoparticles. The data thus are consistent with the two phase cure model discussed above.

\section{Dynamic mechanical analysis data:}

Fig. 5 shows dynamic mechanical data for DEGDA samples containing various concentrations of nanoparticles ranging from 0 to $30 \%$ by weight ( 0 to $8.34 \%$ by volume). The data indicate that the DEGDA systems are heterogeneous with a broad glass transition loss peak and a noticeable shoulder on the low temperature side of the loss tangent dispersion. Peak tan delta values are in the 135 to $145^{\circ} \mathrm{C}$ range. Peak loss modulus values are in the 65 to $75^{\circ} \mathrm{C}$ temperature range as shown in Fig. 6 and Table 1. The glass transition shifts to lower temperatures and the intensity of $\tan \delta$ diminishes as nanoparticles are introduced and their concentration increases. Also the low temperature shoulder shifts to lower temperatures. The storage modulus data indicate that introducing dispersed nano-particles causes only small increases in storage modulus below $\mathrm{Tg}$ but a greater increase in modulus between the neat polymer and the filled polymer are observed above Tg. However, while the plateau modulus above $\mathrm{Tg}$ is similar for the various nanoparticle concentrations, $\tan \delta$ and the loss modulus increase with particle concentration. These results are unexpected. 
For the particle filled composites the Tg loss modulus peak value shifts to lower temperatures and there is a second transition sub-ambient which increases in intensity and shifts to lower temperatures as the particle concentration increases. These changes appear to be consistent with a molecular confinement effect at the particle surfaces [ 18-22 ] associated with the tightly bound short chain silane surface ligand [ 20, 22 ]. The sub $\mathrm{Tg}$ relaxation results from a layer of DEGDA at the interface that is less densely crosslinked than the bulk DEGDA, and therefore has a lower $\mathrm{Tg}$ ( $\alpha$ relaxation). In fact the surface layer appears to be rubbery and the relaxation effects are similar to those described by Gao, et al. [22] who formed epoxy nanocomposites containing $\mathrm{SiO}_{2}$ nanoparticles with a rubbery shell grafted onto the particles. The loss modulus and tan $\delta$ intensity between the $\alpha$ and $\beta$ relaxations is also greater than for neat DEGDA, indicating a gradual change in crosslink density between the polymer at the particle surface and the bulk.

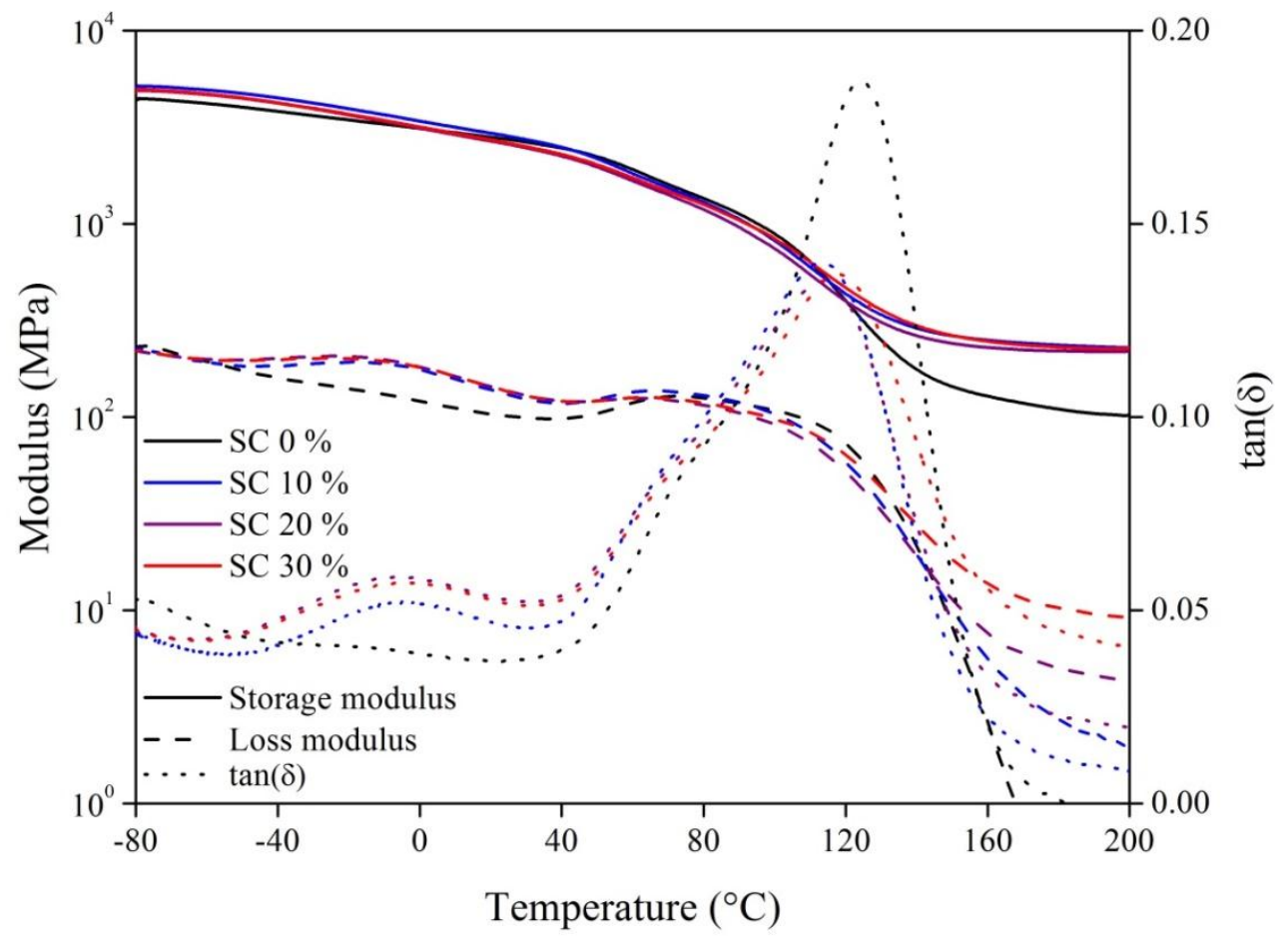

Figure 5: DMA storage modulus, loss modulus and $\tan \delta$ data for neat poly-DEGDA and nano- composites with various nanoparticle concentrations 


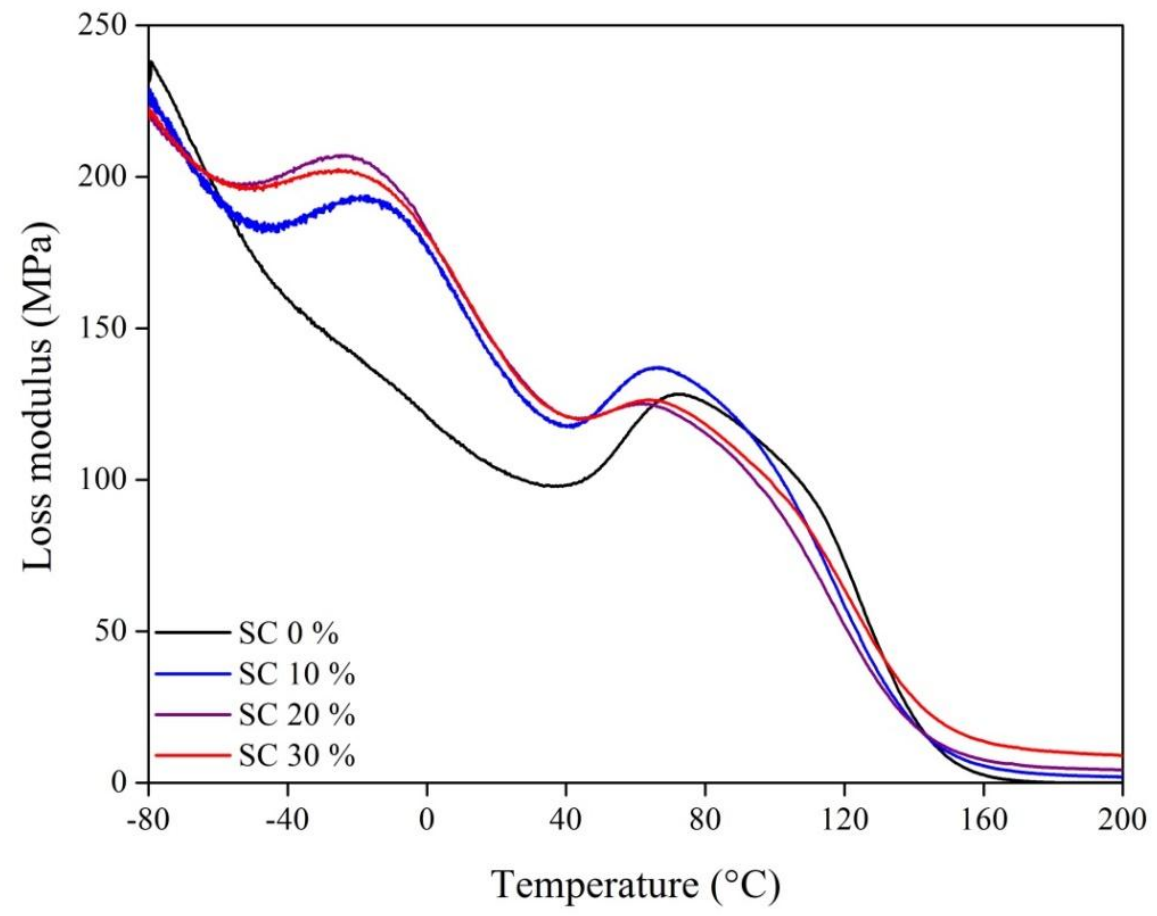

Figure 6: DMA Loss modulus curves (on linear scale) indicating details of the change in position for two transitions that are observed in the composites; the sub $\operatorname{Tg} \beta$ transition is not present in the neat polymer sample

Table 1: Transition temperature variation with concentration of nanoparticles (from loss modulus maxima)

\begin{tabular}{|c|c|c|c|}
\hline \multirow{2}{*}{\multicolumn{4}{|c|}{\begin{tabular}{ccc} 
& \multicolumn{2}{c}{ Alpha - Tg Beta -sub Tg } \\
Wt $\% \quad$ Vol\% & Temperature ${ }^{\circ} \mathrm{C}$
\end{tabular}}} \\
\hline & & & \\
\hline 0 & 0 & 74 & None \\
\hline 10 & 2.3 & 66 & -17 \\
\hline 20 & 5.0 & 64 & -21 \\
\hline 30 & 8.3 & 63 & -22 \\
\hline
\end{tabular}

\section{DEGDA TMA thermal expansion data:}

Poly-DEGDA thermal mechanical analysis (TMA) data shown in Fig. 7 indicate that even though there are varying concentrations of nanoparticles, thermal expansion in the glassy state is similar for the compositions represented with the expansion coefficient slightly greater for the neat DEGDA. An explanation for the similarities in glassy state CTE is not clear. The thermal expansion coefficient above $\mathrm{Tg}$, however, decreases with increasing nanoparticle concentration. While the trend in CTE above $\mathrm{Tg}$ might be expected, it may only reflect the decrease in the polymer mass of the composites. 


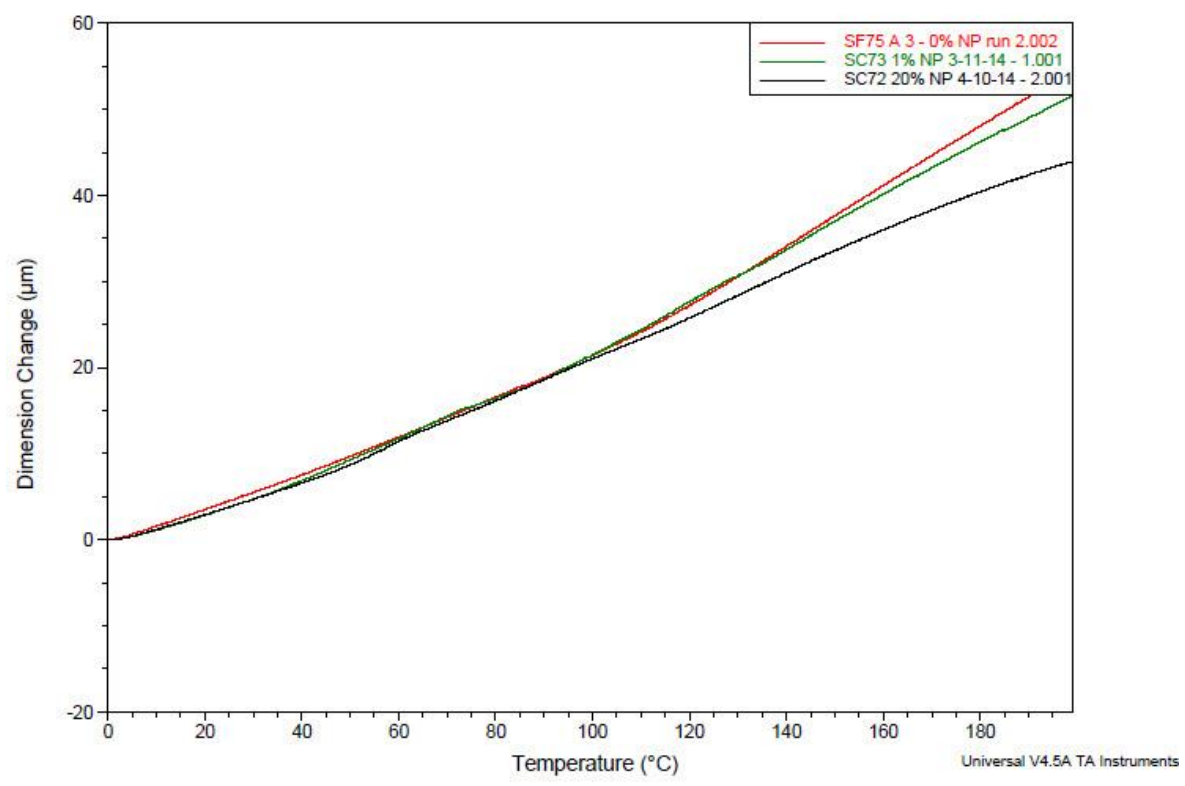

Figure 7: Thermal mechanical analysis (TMA) data for poly-DEGDA as function of nano-particle concentration

Characterizing the interface between layers using SEM; forming a seamless interface between printed layers by limiting $1^{\text {st }}$ pass degree of conversion of polymer:

In order for the composites to function effectively as optical materials the interface between layers must be seamless. This can be controlled by applying the appropriate degree of conversion to the top layer so it is only partially cured. New monomer laid down subsequently will then interdiffuse into the partially cured upper layer. The degree of partial cure should be from $60-80 \%$ as measured by residual heat of reaction by DSC. SEM views of fracture surfaces in Fig. 8 illustrate both "good" (no layers visible) and "bad" (layer lines visible) cases. Layers are $20 \mu \mathrm{m}$ in thickness. Additional reaction of the layer below the top layer occurs on UV exposure of the top layer, so the composite reaches a uniform cure level as it is formed.
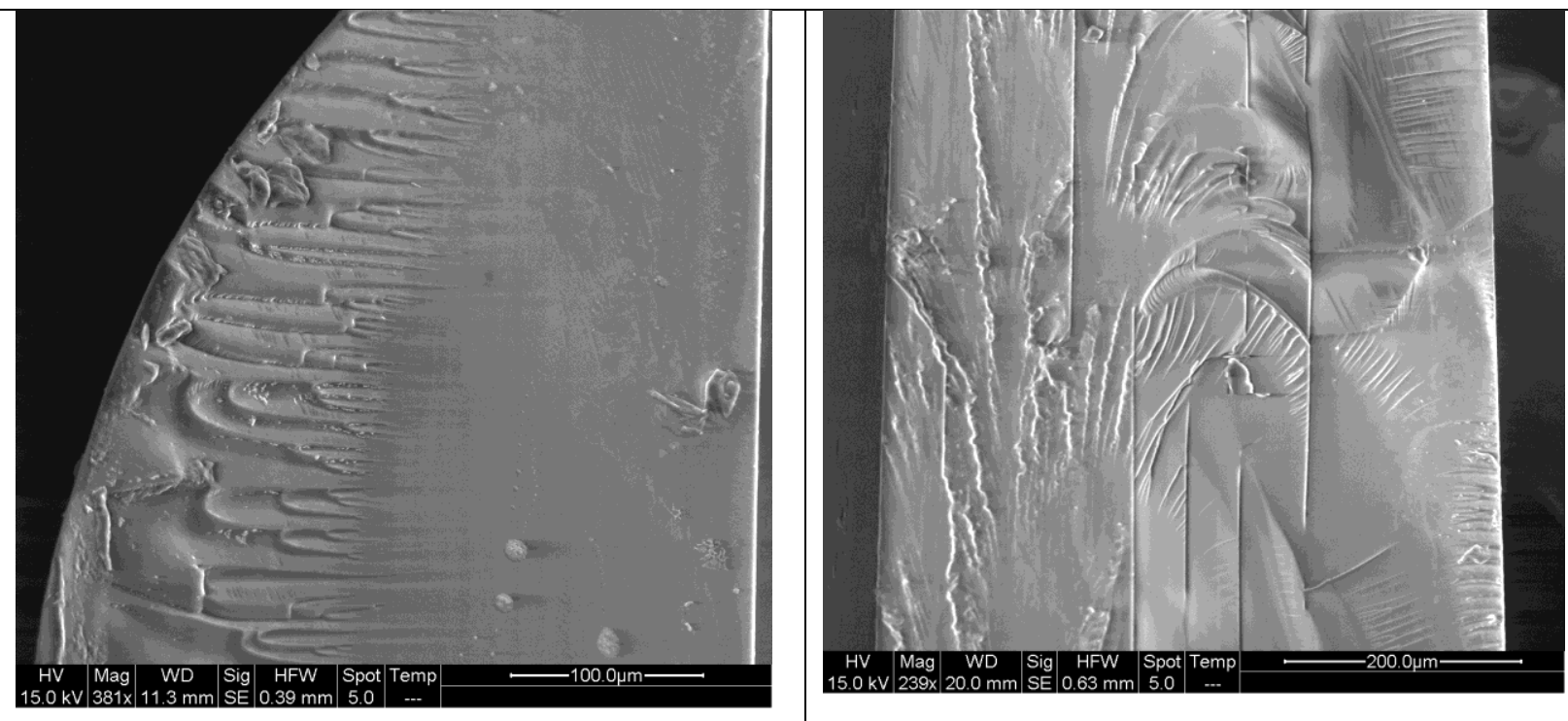
Figure 8: SEM photomicrographs of fracture surfaces of neat DEGDA inkjet printed specimens: 8(a) left showing no layering; 8(b) right -- layered morphology clearly showing defect lines at layer boundaries [ 3 ]

Nanoparticle distribution in printed composites:

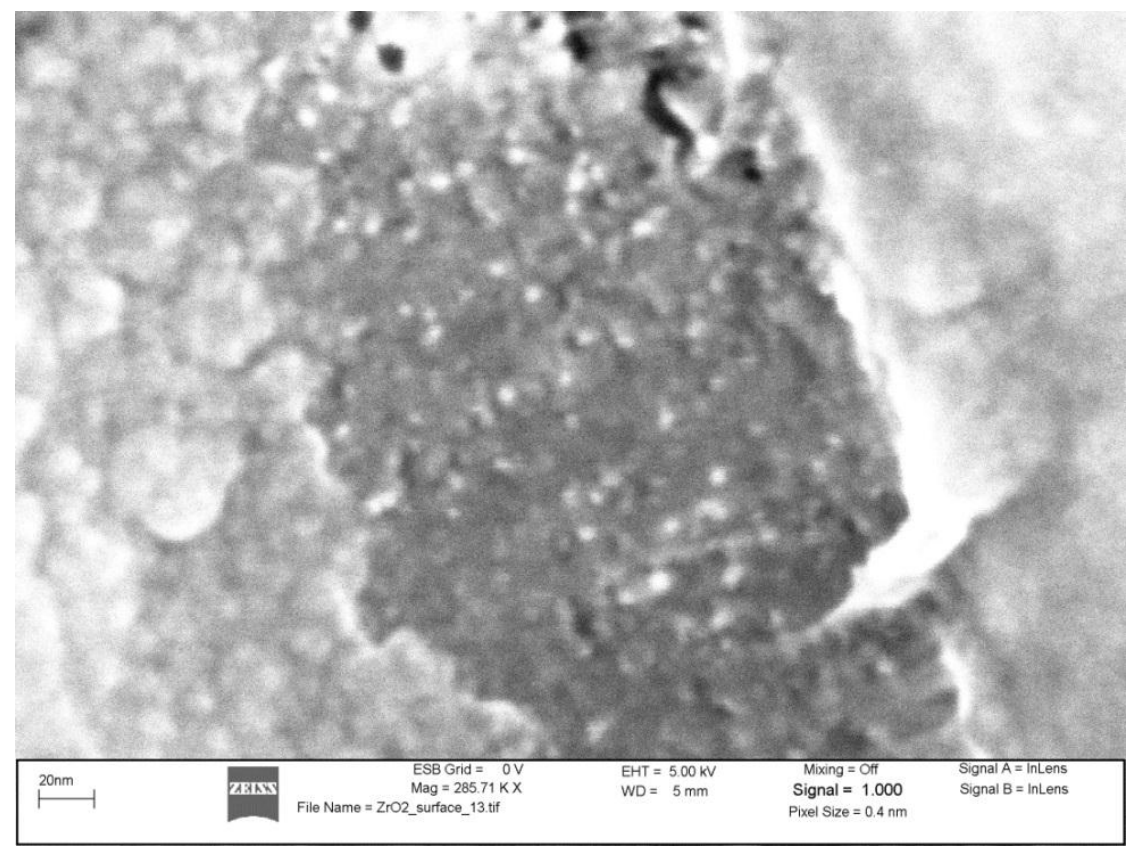

Figure 9: SEM photomicrograph of composite fracture surface of DEGDA nano-composite containing $10 \%$ by weight $\mathrm{ZrO}_{2}$; scale bar at lower left is $20 \mathrm{~nm}$; nanoparticles are well dispersed 


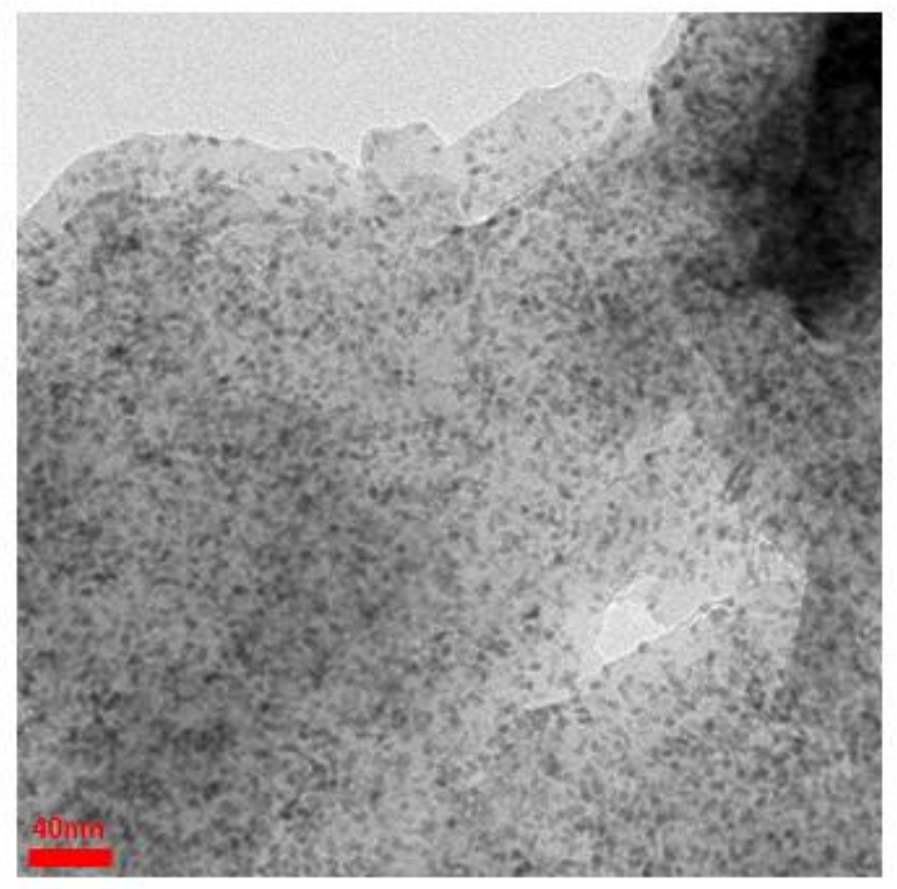

Figure 10: HRTEM of microtomed crossection of a DEGDA composite containing $10 \%$ by weight $\mathrm{ZrO}_{2}$; scale bar at lower left is $20 \mathrm{~nm}$; clearly nanoparticles are well dispersed

SEM and HRTEM photomicrographs of a $10 \%$ by weight $\mathrm{ZrO}_{2}$ nanoparticle composite [Figs. 9 and 10] clearly show well dispersed nanoparticles. An interesting aspect of the photos is the placement of the particles. Apparently the particles are separated by a distance that is about equal to the size of the particles $(7 \mathrm{~nm})$. This indicates that at some higher concentrations the particles may be crowded together enough so as to be, in effect, aggregated. This would be detrimental to optical applications of the nano-composites because it could lead to light scattering and a certain degree of opacity. . However, other researchers have found that up to $50 \%$ wt of $\mathrm{ZrO}_{2}$ can be accommodated in a transparent epoxy nano-composite while still retaining optical clarity [ 19 ].

\section{Inkjet printed multilayer gradient nano-composite:}

The type of data generated in the cure study was used to form gradient nano-composite films by inkjet printing. UV absorbance data for an example of a 30 layer linear gradient film is presented in Fig. 11. The $333 \mathrm{~nm}$ UV absorbance profile taken from left to right along the length of the sample is shown. The linear optical gradient along the length of the film is created by a linear decrease in nanoparticle concentration from 25 to zero \% wt $\mathrm{ZrO}_{2}$. 


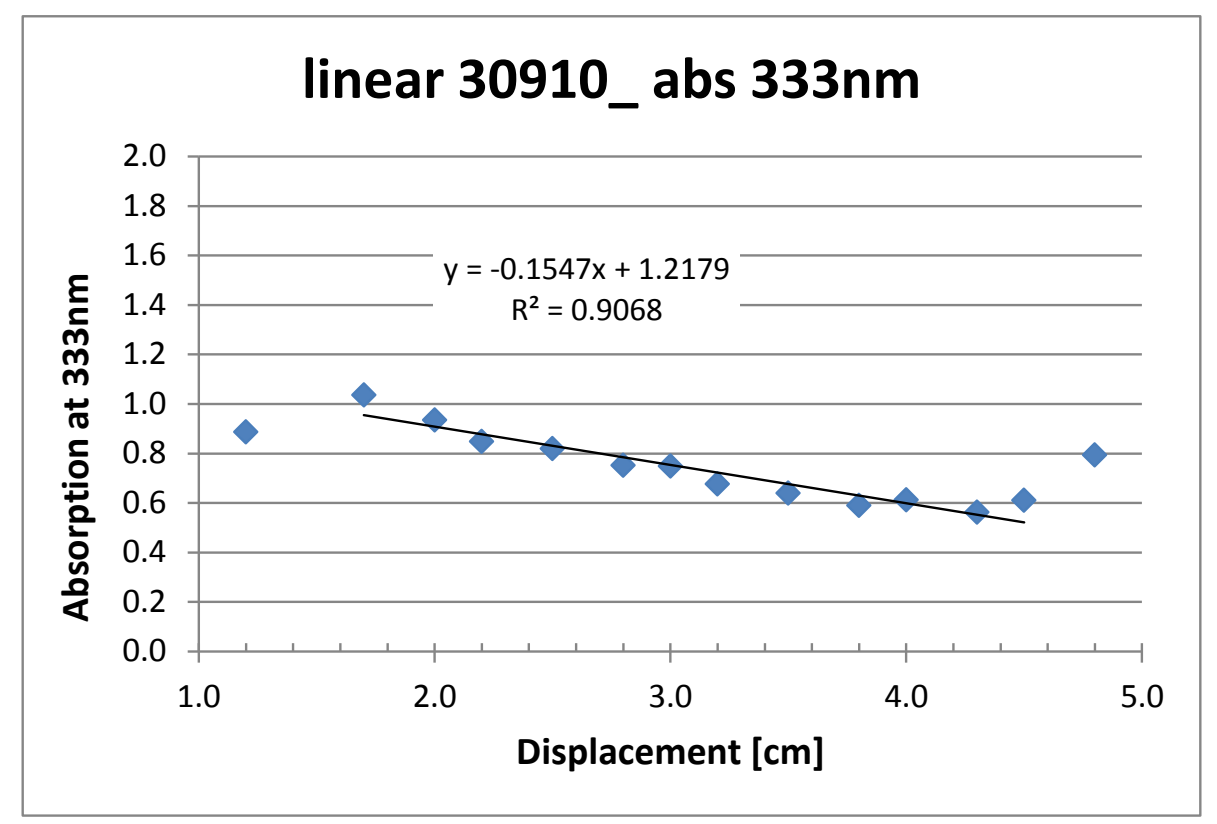

Figure 11: 30 layer DEGDA optical gradient film printed with decreasing $\mathrm{ZrO}_{2}$ concentration from 25 to zero \% wt from left to right along the $5 \mathrm{~cm}$ length; data show that UV absorption at $333 \mathrm{~nm}$ decreases linearly in proportion to $\mathrm{ZrO}_{2}$ concentration

\section{CONCLUSIONS}

The following is a summary of the major findings of this research:

The UV photopolymerization to form poly-DEGDA nano-composites containing various amounts of $7 \mathrm{~nm} \mathrm{ZrO}$ nanoparticles was studied with respect to variations in UV light exposure. The degree of conversion achieved vs light intensity was measured by DSC for various compositions ranging from 0 to $30 \%$ by weight ( 0 to $8.3 \%$ by volume) of nanoparticles. In all cases it was found that including nanoparticles in the monomer significantly increased the conversion achieved. This was attributed to the influence of a silane surface treatment on the nanoparticles. However, as the particle concentration increased the differences in conversion for a given UV exposure level diminished.

In order for the composites to function effectively as optical materials the interface between layers must be seamless. The degree of cure must be controlled to between 60 and $80 \%$ by applying the appropriate light dosage to the top layer so it is only partially cured. New monomer laid down subsequently will then more readily interdiffuse into the partially cured upper layer.

SEM and TEM photomicrographs of composites showed that the particles were well dispersed, but at higher concentrations crowding of the particles could occur, so as to cause the particles to aggregate sufficiently to hamper light transmission through the composites.

DMA characterization indicated that the effect of the nanoparticles in the glassy state was significant. In the nanoparticle filled materials a sub $\operatorname{Tg} \beta$ relaxation was evident that increased in intensity with increased particle concentration. The presence of nanoparticles in the polymer caused a decrease in intensity of the $\mathrm{Tg}(\alpha)$ relaxation as well as a shift in the $\mathrm{Tg}$ loss maximum to lower temperatures. The observed dependencies of both the $\alpha$ and $\beta$ relaxations on particle concentration are interpreted as resulting from the presence of an interfacial layer that has a much lower $\mathrm{Tg}$ because it is rubbery. 
Chemical conversion data such as those presented here provide information suitable for determining the appropriate light exposure for creating defect free gradient nano-composites with seamless interfaces between layers. Having this data enables us to use inkjet printing as an effective method for producing gradient nano-composites which are customized for specific optical applications.

\section{ACKNOWLEDGEMENTS}

This research was sponsored in part by the US National Science Foundation (NSF) via grant CMMI-1069295. The NSF program manager was Dr. Mary Toney. The authors are grateful for the support from NSF. Partial support also was provided by the Oregon Nanoscience and Microtechnologies Institute (ONAMI); Robert D."Skip" Rung, President and Executive Director. The authors acknowledge use of the excellent nano-science analytical facilities of the University of Oregon Center for Advanced Materials Characterization in Oregon (CAMCOR): (camcor.uoregon.edu.). The program sponsors had no role in the research.

\section{REFERENCES}

1. C. Check, R. Chartoff, S.Chang, European Polymer Journal, 70, (2015)166-172

2. R.P. Chartoff, C Check, U.S. Patent Application No. 13/944,793, Filed July 17, 2013

3. J.G. Kloosterboer, Makromol. Chem.: Makromol. Symp, 224, (1989) 223

4. R.P. Chartoff, C Check, U.S. Patent Application No. 13/944,793, Filed July 17, 2013

4. H.M.J. Boots, J. G. Kloosterboer, G. M. M. Van de Hei, R. B. Pandey, British Polym. J, 17, (1985) 219.

5. J.G. Kloosterboer, G. F. C. M. Lijten, H. M. Boots, J. Makromol. Chem, Macromolecular Symposia, 24, (1989) 223.

6. J.G. Kloosterboer, G. F. C. M. Lijten, C. P. G. Zegers, ACS Polymeric Materials Science and Engineering Preprints, 60 (1989) 122

7. R Chartoff, Journal of Thermal Analysis and Calorimetry, 85 (1), (2006) 213-217

8. J E. Dietz and N. A. Peppas, Polymer, 38 (1997) 3767.

9. J. Pavlinec and N. Moszner, J. Appl. Polym. Sci, 89 (2003) 579.

10. E Andrzejewska, Prog. Polymer Science, 26, ( 2001) 605

11. G. Williams, et.al., "Synthesis, capping and dispersion of nanocrystals" International Patent Application: No. WO 2011/133228 A2, Filed 23 April 2011

12. H.-H. Lee, K.-S. Chou1, K.-C. Huang, "Inkjet printing of Nanosized Silver Colloids", Nanotechnology, 16, (2005) 2436-2441

13. Q. Li, and J. A. Lewis, "Nanoparticle Inks for Directed Assembly of Three-dimensional Periodic Structures”, Adv. Mater., 15 (19), (2003)1639

14. B Cappi, J Ebert, R Telle, "Rheological Properties of Aqueous $\mathrm{Si}_{3} \mathrm{~N}_{4}$ and $\mathrm{MoSi}_{2}$ Suspensions Tailor-Made for Direct Inkjet Printing", J. Am. Ceram. Soc., 94 (10), (2011) 111-116 
15. B. Derby, " Inkjet Printing of Functional and Structural Materials:Fluid Property

Requirements, Feature Stability, and Resolution", Annual Rev. Mater. Res., 40, (2010) 395-414

16. H-G Woo, B-H Kim, M-S Cho, D-Y Kim, Y-S Choi, Y-C Kwak, H-S Ham, D P Kim, T S Hwang, Bull. Korean Chem. Soc., 22 (12), (2001)1337

17. H-G Woo, LY Hong, S-Y Kim, Y-S Choi, S H Park, S J Song, H-S Ham, Bull. Korean Chem. Soc., 16 (8), (1995) 775

18. C. J. Ellison, J. M. Torkelson, Nature Materials, 2, (2003) 695-700

19. P. Akcora S. K. Kumar, V. G. Sakai, Y. Li, B. C. Benicewicz, L. S. Schadler, Macromolecules, 43, (2010) 8275-8281

20. P. Tao, Y. Li, R. W. Siegel, L. S. Schadler, J. Appl. Polym. Sci, DOI: 10.1002/APP.39652, (2013) 1-9.

21. J. Choi, M. J. A. Hore, N. Clarke, K. I. Winey, R. J. Composto, Macromolecules, 47, (2014) 2404-2410

22. J. Gao, J, Li, B.C. Benicewicz, S. Zhao, H. Hillborg, L. S. Schadler, Polymers, 4, (2012) $187-210$ 